\title{
Porphyrin as a Versatile Visible-Light-Activatable Organic/Metal Hybrid Photoremovable Protecting Group
}

Adiki Raja Sekhar ${ }^{1}$, Youhei Chitose, ${ }^{2,3}$ Jiří Janoš $^{4}$, Sahar Israeli Dangoor ${ }^{5}$, Andrea Ramundo², Ronit Satchi-Fainaro ${ }^{5}$, Petr Slavíček ${ }^{4}$, Petr Klán ${ }^{2, *}$, Roy Weinstain ${ }^{1, *}$

${ }^{1}$ School of Plant Sciences and Food Security, Faculty of Life Sciences, Tel-Aviv University, Tel-Aviv 6997801, Israel. ${ }^{2}$ Department of Chemistry and RECETOX, Faculty of Science, Masaryk University, Kamenice 5, 625 00, Brno, Czech Republic. ${ }^{3}$ Basic Chemistry Program, Graduate School of Advanced Science and Engineering, Hiroshima University, HigashiHiroshima 739-8526, Japan. ${ }^{4}$ Department of Physical Chemistry, University of Chemistry and Technology, Technická 5, 16628 Prague 6, Czech Republic. ${ }^{5}$ Department of Physiology and Pharmacology, Sackler School of Medicine, Tel Aviv University, 69978 Tel Aviv, Israel.

\section{Introduction}

Photoremovable protecting groups (PPGs), also known as photocaged compounds, are linked to a molecule of interest to mask its chemical or biological activity. Upon irradiation at a specific wavelength, the PPGs are tracelessly removed, allowing non-invasive spatio-temporal control over the release of free molecules with a high degree of chemoselectivity in complex chemical and biological environments. Consequently, they represent one of the main contemporary applications of photochemistry in diverse research areas, ranging from chemistry and material science to biology and medicine. ${ }^{1,2,3,4}$

Since their introduction more than 50 years ago, ${ }^{5,6}$ dozens of PPG types have been developed. ${ }^{7}$ 8 Their fundamental, mutually exclusive classification differentiates between organic and transition-metal complex PPGs (Fig. 1a). Transition-metal-free (organic) PPGs possess an organic chromophore and liberate molecules via the cleavage of a covalent bond. ${ }^{7,8}$ This large class of PPGs encompasses a wide range of molecular scaffolds, from the established nitroaryl and coumarin groups to the more recent, visible-light absorbing BODIPY and cyanine groups. ${ }^{7}$ ${ }^{8}$ In contrast, metal-complex PPGs consist of a central metal atom or ion surrounded by ligands as part of their chromophores. The complexes have unique ground- and excited-state properties, arising from the nature of the central metal, the ligands, and specific interactions between them. The metal coordination center serves as a bonafide reaction center in all metalcomplex PPGs reported to date, and ligand exchange is the ultimate liberation step, succeeding a photoinitiated metal-to-ligand, ligand-to-metal, or ligand-to-ligand charge-transfer transition. ${ }^{7,8,9,10}$ Notable examples in this class include cob(III)alamins, and ruthenium and rhodium complexes. ${ }^{9,} 10$

Over the decades, the conceptual dichotomy between these two PPG classes has been maintained, being translated into essentially non-converging development paths. To date, efforts to exploit the distinctive properties of organic and metal-complex photoactivatable systems within a single structure have primarily focused on either linking the established metalfree PPGs to metal complexes, metal surfaces, or metal nanoparticles or, on the other hand, on the conjugation of metal-complex PPGs to organic chromophores (antennas). ${ }^{8} \mathrm{We}$ considered 
a hybrid class of PPGs, which allows (but do not require) the incorporation of a metal (ion) as a part of the chromophore and releases leaving groups through the photoinduced cleavage of covalent bonds (Fig. 1b). We reasoned that blurring the traditional dividing line between these two PPG classes would provide access to a yet untapped chemical space of this new type of PPGs.

To evaluate this concept, we first repurposed the ubiquitous ligand porphyrin as an independent PPG. We then show that the functional separation of the metal-binding chromophore and the site of leaving group release can be leveraged to fine-tune its spectroscopic and photochemical properties by simply introducing metal ions into the porphyrin core. The release of leaving groups from the free-ligand as well as metal-containing porphyrin derivatives was studied by both steady-state and transient-absorption spectroscopies, and the results were supported by quantum-chemical calculations. We thus provide a detailed description of the photoreaction mechanism, laying the foundations for further expansion and optimization of such hybrid-class PPGs.

\section{Results and Discussion}

\section{Metal-Free Porphyrin PPGs}

The design of a porphyrin-based PPG was inspired by the recent emergence of the meso-methyl functionalized boron dipyrromethene (BODIPY) group. ${ }^{11}{ }^{12}$ Hückel molecular orbital predictions showed that BODIPY PPGs could release a leaving group thanks to the negative charge increase at the meso position upon excitation. ${ }^{13}$ The structurally related porphyrin scaffold has four such positions, which exhibit a similar electronic behavior upon excitation to the first excited state (see Supplementary Section 4).

The synthesis of an appropriate porphyrin core was accomplished by MacDonald acidcatalyzed condensation (Fig. 2a). ${ }^{14}$ The ortho-unsubstituted phenyl group of $\mathbf{1}$ and the aliphatic aldehyde in 2 facilitated a scrambling mechanism ${ }^{15,16}$ that gave rise to the formation of all possible porphyrin combinations (Supplementary Fig. 1), including the key A3B-type derivatives 3. Subsequent alcohol deprotection provided the metal-free meso-methylporphyrin 4. The hydroxyl group in $\mathbf{4}$ is a poor nucleophile; its reactions with chloroformate and isocyanate were not successful. However, it was found to be sufficiently nucleophilic to react with $O$-acylisourea derivatives or mesyl chloride, allowing its in-situ displacement with nucleophiles such as $p$-nitrobenzoic acid (PNBA) or $N, N$-dimethylaminopyridine (DMAP) to give the final derivatives $\mathbf{5}$ and $\mathbf{6}$, respectively.

The photophysical and photochemical properties of 5 and $\mathbf{6}$ in DMSO or methanol solutions are summarized in Table 1. Analogous to metal-free porphyrins, ${ }^{17}, 18$ the $S_{0} \rightarrow S_{2} / S_{1}$ electronic transitions (Q bands) were found in the range of 500-650 $\mathrm{nm}$, and very intense absorption bands with the maxima $\lambda_{\max }^{\text {abs }}$ in the blue region were assigned to the $\mathrm{S}_{0} \rightarrow \mathrm{S}_{4} / \mathrm{S}_{3}$ transitions (Soret bands; Fig. 2b and Supplementary Fig. 2). ${ }^{19}$ The photorelease of leaving groups (LG) from 5 and 6 was monitored by LG appearance (HPLC) and starting material disappearance (absorption spectroscopy; Fig. 2c and Supplementary Fig. 3). The LGs were liberated with chemical yields of $>70 \%$ and photodecomposition quantum yields $\left(\Phi_{\text {dec }}\right.$ ) of $\sim 0.002$ upon 
irradiation at $410 \mathrm{~nm}$. It is worth noting that the large molar absorption coefficients at the irradiation wavelength $\left(\lambda_{\text {irr }}\right)$ are responsible for very high uncaging cross sections ( $\left.\Phi_{\mathrm{dec}} \varepsilon_{\max }\left(\lambda_{\mathrm{irr}}\right)\right)^{7,8}$ of $>650 \mathrm{M}^{-1} \mathrm{~cm}^{-1}$.

The structural and physico-chemical properties of porphyrin allow for a high degree of modularity in its application as a PPG. For example, the photorelease of an LG from $\mathbf{6}$ can be achieved by irradiation to its different absorption bands in the visible region (Fig. $2 \mathrm{~d}$ and Supplementary Fig. 4). The fluorescence ( $\Phi_{\mathrm{F}}$; in the range of $420-560 \mathrm{~nm}$ ) was found to be wavelength-independent. In addition, some structural modifications of the basic scaffold can be implemented without compromising its functionality. Substituents on the meso-phenyl groups generally modulate the electronic properties of the porphyrin chromophore only to a small extent. ${ }^{20}$ Indeed, derivatives 7 and 8, featuring the 4-methoxyphenyl or 4-Opropargylphenyl groups (Fig. 2a), exhibited spectroscopic and photochemical properties similar to those of 5 (Table 1). The lower $\Phi_{\text {dec }}$ found for 8 can be explained by the increased rotational freedom of the molecule. ${ }^{21}$ Nevertheless, the alkyne groups in $\mathbf{8}$ provided convenient reaction sites for further functionalization via a copper-click reaction (Supplementary Fig. 5). An additional level of modularity emerges from the multiple meso positions in the porphyrin scaffold. Taking advantage of this structural feature, $\mathrm{A}_{3} \mathrm{~B}-, \mathrm{A}_{2} \mathrm{~B}_{2}$ - and $\mathrm{B}_{4}$-type porphyrin PPGs bearing $N_{\alpha}$-Boc- $L$-tryptophan as a model LG were synthesized (11-13, Fig. 2e). The number of equivalents of $L G$ released was 2-fold from the $\mathrm{A}_{2} \mathrm{~B}_{2}$-type 12 and 2.9-fold from the $\mathrm{B}_{4}$-type 13 when compared to that from the $\mathrm{A}_{3} \mathrm{~B}$-type 11 (Fig. 2e), probably involving an enhanced non-productive photodegradation of the chromophore during the sequential release of multiple LGs.

To evaluate the functionality of the meso-methylporphyrin group in a complex environment, we synthesized derivatives bearing the tubulin assembly inhibitor indibulin or the antifolate agent methotrexate (MTX) as LGs (9 and 10, respectively, Fig. 2a). Compound 9 released indibulin upon irradiation, but it was found to be thermally unstable in a PBS buffer (Supplementary Fig. 6), unlike a recently reported coumarin-caged indibulin that was effectively applied in cultured cells. ${ }^{22}$ Conversely, $\mathbf{1 0}$ was thermally stable, and its photoactivation was successfully conducted upon $545 \mathrm{~nm}$ irradiation because MTX itself absorbs and decomposes when photolyzed at $410 \mathrm{~nm}$ (Supplementary Fig. 6). Thus, using 10, we carried out a cellular evaluation in cultures of the murine 4T1 mammary carcinoma cell line. Fluorescence microscopy indicated that the molecule is taken up into cells and appears to be localized in punctate structures that do not co-localize with lysosomal compartments (Fig. 2f,g). Maximum intracellular signal was observed after 2-h preincubation (Supplementary Fig. 6). Cell viability measurements (MTT assay) demonstrated that compound $\mathbf{5}$, bearing a nontoxic $\mathrm{LG}$, has low light-dependent toxicity $\left(\mathrm{IC}_{50}=57.8 \mu \mathrm{M}\right.$, Figure $2 \mathrm{~h}$ ), which was attributed to the photodynamic effect (in accord with $\Phi_{\Delta}$; Table 1 ). In comparison, 10 is $\sim 15$-times more toxic than 5 when exposed to light $\left(\mathrm{IC}_{50}=4.0 \mu \mathrm{M}\right)$. Collectively, the above results confirm the viability of meso-methylporphyrin as a versatile PPG scaffold. 


\section{Metal-Containing Porphyrin PPGs}

The ability of porphyrin to chelate a large number of metal cations provides an unprecedented approach to manipulating the properties of this PPG. We synthesized complexes of $\mathbf{6}$ chelating metal cations with distinct electronic configurations (i.e., $\mathrm{Zn}(\mathrm{II}), \mathrm{Pd}(\mathrm{II}), \mathrm{Cu}(\mathrm{II})$, or $\mathrm{Ni}(\mathrm{II})$ in compounds 6-Zn, 6-Pd, 6-Cu, and 6-Ni respectively; Fig. 3a). A single-crystal X-ray diffraction analysis of $\mathbf{6 - Z n}$ is shown in Supplementary Fig. 7. The photophysical and photochemical properties of metal porphyrins are summarized in Table 1. The metalcomplexes 6-Pd, 6-Cu, and 6-Ni exhibited $\lambda_{\max }^{\text {abs }}$ values similar to that of $\mathbf{6}$ but with significantly lower molar absorption coefficients $\left(\varepsilon_{\max }\right)$ and fluorescence quantum yields $\left(\Phi_{\mathrm{F}}\right)$ (Supplementary Fig. 2). In comparison, derivative 6-Zn possessed a 9-nm red-shifted $\lambda_{\max }^{\text {abs }}$ value, a slightly higher $\varepsilon_{\max }$, and a shorter fluorescence lifetime. Upon irradiation at $410 \mathrm{~nm}$ in DMSO solutions, the release of DMAP was observed from 6-Zn and 6-Pd in $74 \%$ chemical yields. However, no photorelease was observed for derivatives 6-Cu and 6-Ni (Fig. 3c and Supplementary Fig. 3). The photodecomposition quantum yield $\Phi_{\text {dec }}$ of 6-Zn was $\sim 12$-times higher than that of the free-ligand derivative 6 (Table 1), resulting in an extraordinarily high uncaging cross section of $\Phi_{\mathrm{dec}} \varepsilon_{\max }=9634 \mathrm{M}^{-1} \mathrm{~cm}^{-1}$, one of the highest to be reported to date. ${ }^{7}$ ${ }^{8}$ The photodecomposition of 6-Pd was $\sim 6$-times less efficient than that of $\mathbf{6}$.

We also examined excitation wavelength $\left(\lambda_{\text {exc }}\right)$ dependence of fluorescence and photorelease efficiencies. Analogous to compound $\mathbf{6}, \Phi_{\mathrm{F}}$ was found practically $\lambda_{\mathrm{exc}}$ independent for 6-Zn in the range of 420-560 $\mathrm{nm}$ (Supplementary Table 1). More importantly, the quantum yield values of DMAP release ( $\Phi_{\mathrm{r}}$; HPLC) from $\mathbf{6 - Z n}$ were found within a narrow range of 0.016 , 0.008 , and 0.007 when irradiated at Soret and Q bands at $\lambda_{\text {irr }}=427,560$, and $600 \mathrm{~nm}$, respectively (Supplementary Table 3), therefore, the entire visible range can be used for excitation. The $\Phi_{\mathrm{r}}$ values are lower than those of $\Phi_{\text {dec }}$ because the maximum chemical yield of LG release reached $74 \%$ (i.e., $26 \%$ of excited molecules are decomposed unproductively; Table 1).

\section{Mechanistic Studies}

A detailed mechanistic study was carried out to understand the photochemistry of LG photorelease from meso-methylporphyrin derivatives and support the further development of these hybrid-class PPGs.

Photoproducts. A solvent-capture photoproduct 4a was formed upon irradiation of $\mathbf{6}$ in degassed DMSO/water, along with porphyrin-meso-carbaldehyde $\mathbf{1 4}$ and other unidentified photoproducts (Supplementary Fig. 8). The formation of 4a pointed either to a meso-methyl cation intermediacy, analogous to that observed in meso-methyl BODIPY PPGs, ${ }^{23}$ or to an $\mathrm{S}_{\mathrm{N}} 2-$ like process. Aldehyde $\mathbf{1 4}$ was most probably formed upon oxidation by DMSO (Supplementary Fig. 8c), as previously reported in some coumarin PPGs. ${ }^{24}$ Significantly higher amounts of the solvent-capture photoproduct compared to that of $\mathbf{6}$ were found upon irradiation 
of 6-Zn in degassed DMSO/water or methanol solutions (Supplementary Fig. 9), implying a profound effect of the metal type on the porphyrin PPG photochemistry. ${ }^{25,26}$

Effects of Oxygen. Monitoring the photodegradation of $\mathbf{6}$ and 6-Zn at short time intervals and low-intensity light $\left(410 \mathrm{~nm}, 3.2 \mathrm{~mW} \mathrm{~cm}^{-2}\right.$ ) revealed a delay in the onset of photolysis when performed in aerated compared to degassed DMSO or DMSO/water solutions (Fig. 4a and Supplementary Fig. 10). In contrast, the photodegradation of 6 in methanol was observed only in the absence of oxygen (Fig. 4b). In addition, the photoreaction of 6 in aerated methanol proceeded smoothly in the presence of a singlet oxygen quencher (furfuryl alcohol, Fig. 4b and Supplementary Fig. 11), which excluded singlet oxygen as a photorelease mediator. These results suggest that initial ${ }^{1} \mathrm{O}_{2}$ generation (see the Jablonski diagram, Fig. 4c) by the excited porphyrin outcompetes the photorelease, which is in accord with the measured quantum yields of both processes ( $\Phi_{\Delta}$ is $\sim 2$ orders of magnitude higher than $\Phi_{\text {dec }}$, Table 1). Once ground state oxygen is partially depleted from the immediate environment, the photorelease can proceed efficiently. Thus, the unusual course of photochemistry observed in aerated DMSO solutions most probably reflects the initial reaction (depletion) of singlet oxygen, generated by excited porphyrin, with DMSO molecules, ${ }^{27}$ which does not occur in methanol. A similar effect of local molecular oxygen depletion on a photoreaction has recently been reported for the photoactivation of gold(I) arylethynyl complexes phosphorescence. ${ }^{28}$ The ability of groundstate oxygen to quench the photorelease process also suggests that the reaction proceeds from the triplet excited state.

The singlet oxygen production efficiencies of $\Phi_{\Delta}=0.16,0.25$, and 0.23 , determined for $\mathbf{6}$, 6Zn and 6-Pd, respectively, in methanol, and relatively low fluorescence quantum yields measured for these compounds (Table 1) were used to estimate the upper limit of ISC quantum yield $(<\sim 0.7)$. The relatively low values of $\Phi_{\text {dec }}(<0.02)$ are approximately 2 orders of magnitude lower than those of $\Phi_{\Delta}$; therefore, the production of singlet oxygen is the major competing pathway to the LG release. Fortunately, it was found that singlet oxygen is not primarily responsible for the chemical degradation of these PPGs (e.g., excitation of a singlet oxygen generator, rose bengal, at $\lambda_{\text {irr }}=545 \mathrm{~nm}$ in the presence of 6 in aerated methanol did not lead to its decomposition, Fig. 4b).

Transient Spectroscopy. The excited-state radiative and non-radiative deactivation pathways for metal-free and metal-containing model porphyrin derivatives (tetrakis(4carboxyphenyl)porphyrin, see Supplementary Section 3), which do not bear an LG, and for porphyrin PPGs 6, 6-Zn, 6-Pd, and 6-Cu were studied by femtosecond (fs) and nanosecond (ns) transient absorption (TA) spectroscopies. We found that neither the absence of an LG in the meso-methyl position nor the presence of 4-carboxyl groups on meso-phenyl substituents in the model derivatives had any significant effect on the spectroscopic behavior of all studied compounds.

The fs-TA spectra, evolution associated spectrum (EAS) components, and their relative populations for porphyrins 6, 6-Zn, 6-Pd, and 6-Cu in DMSO are shown in Supplementary Fig. 12, and the data are summarized in Table 2. Global analyses identified four evolution- 
associated spectra (EAS) components for $\mathbf{6}$ and $\mathbf{6}-\mathbf{Z n}$. As in the case of the model porphyrins, we can attribute the observed components (EAS1, EAS2) to the transitions occurring within Q bands ( $k_{\mathrm{Q}}^{1}$ and $k_{\mathrm{Q}}^{2}$, Table 2 ), such as vibrational cooling or conformational relaxation. For example, it has been reported that conformational relaxation in tetraphenylporphyrin occurs in the $\sim 100$ ps time scale. ${ }^{29}$ Analogous to the kinetics of the model compounds (see Supplementary Section 3), and also reported for other porphyrin derivatives, ${ }^{30,31,32,33,34,35}$ the transition between EAS3 and EAS4 was attributed to singlet-triplet intersystem crossing (ISC; $k_{\text {ISC }}=1.7 \times 10^{8} \mathrm{~s}^{-1}$ and $2.2 \times 10^{8} \mathrm{~s}^{-1}$ for $\mathbf{6}$ and $\mathbf{6}-\mathbf{Z n}$, respectively). A much faster ISC ( $k_{\mathrm{ISC}}=$ $1.6 \times 10^{10} \mathrm{~s}^{-1}$ ) and only two EAS components were observed for 6-Pd, which is related to the heavy-atom effect of the Pd atom. A simplified Jablonski diagram depicting all fundamental photophysical and photochemical processes relevant to the photochemistry of $\mathbf{6 , 6 - Z n}$, and 6Pd is shown in Fig. 4c.

The fs-TA spectra of a $\mathrm{Cu}(\mathrm{II})$ derivative 6-Cu displayed a narrow band at $\sim 440 \mathrm{~nm}$ in the ps time scale (EAS1), but the rapid subsequent processes made it difficult to monitor all evolution steps. The EAS1 $\rightarrow$ EAS2 transition $\left(k=6.7 \times 10^{11} \mathrm{~s}^{-1}\right)$ was followed by the appearance of a very weak transient signal in the region of 440-520 nm (EAS3), and it was accompanied by an artifact monitored at 530-700 nm, attributed to the signal of the solvated electron. ${ }^{36}$ The previous interpretations of analogous data assigned the first two transients as a tripdoublet ${ }^{2} \mathrm{~T}$ state, a ${ }^{2}[\mathrm{~d}, \mathrm{~d}]$ exciplex state, formed by the ligation with $O$-coordinating solvents, respectively. ${ }^{36,37,38}$ Consequently, we conclude that the uncaging process from 6-Cu cannot compete with a very fast radiationless decay of the productive excited state.

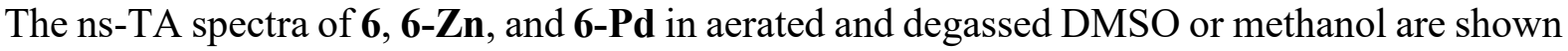
in Supplementary Fig. 13, and the data are summarized in Table 2. The triplet lifetimes of these compounds were found to be $\sim 6$-times shorter in aerated than degassed DMSO due to quenching of the triplet state by oxygen. Shorter triplet lifetimes in 6-Pd are related to a faster triplet-singlet ISC (heavy atom effect). ${ }^{39,40,41,42}$ Very similar kinetic data were obtained in methanol solutions (Table 2); all rate constants were only slightly faster than those obtained in DMSO. It is worth mentioning that the triplet lifetimes of porphyrin PPGs show a clear correlation with their photodecomposition quantum yields $\left(\Phi_{\mathrm{dec}}\right)$, further indicating that the photorelease of DMAP proceeds via the triplet excited state.

Quantum Chemical Calculations and the Proposed Release Mechanism. Based on the experimental observations and ab initio calculations and simulations presented in Supplementary Section 4, we propose the photoreaction mechanism involving release of an LG to form the carbocation in the triplet state that subsequently intersystem crosses and reacts with a nucleophile, is proposed in Figure 4d. The nucleophilic substitution of a leaving group was calculated on simplified model compounds (Supplementary Section 4); it was found to be a slightly endergonic reaction for the reaction with water under standard conditions. Porphyrin derivatives are excited into the highly absorbing Soret band. This state is typically located as a third or fourth excited state, ${ }^{43,44}$ except for the $\mathrm{Cu}$-containing derivative possessing a large number of weakly absorbing states below the Soret band. The dynamical simulations predict an ultrafast internal conversion, completed in $\sim 100$ fs. The porphyrins in the $S_{1}$ state then 
deactivate primarily via intersystem crossing; the importance of coupling between the vibrations and electronic transitions has previously been emphasized. ${ }^{44}$ We propose that the majority of the product is formed from a triplet excited porphyrin derivative via an $\mathrm{S}_{\mathrm{N}}$ 1-like mechanism. The calculated activation Gibbs energies in the triplet state for the formation of the corresponding carbocation (in the rate-limiting step) varies in the range of $0.5-1 \mathrm{eV}$; this corresponds to the rate constants of up to $10^{5} \mathrm{~s}^{-1}$. A long triplet lifetime is, therefore, critical for the reaction. The triplet carbocation formed is strongly stabilized by resonance. Note that the energy barriers are similar for the cleavage from the $\mathrm{S}_{1}$ electronic state, which has, however, a much shorter lifetime. The above mechanism is operative for $\mathbf{6}, \mathbf{6 - Z \mathbf { n }}$, and $\mathbf{6 - P d}$, and the release efficiency is controlled primarily by the triplet state lifetime. The situation is different for 6-Cu, where the d- electrons of the metal strongly interact with the porphyrin ring. As a result, its excited-state deactivation is very short (tens of picoseconds). ${ }^{36,45,46}$ In the literature, the fast process is usually interpreted as IC to the ${ }^{2} S_{1}$ state $\left(S_{1}\right.$ state of the porphyrin with a doublet state of $\mathrm{Cu}^{2+}$ ), accompanied by extremely fast ISC to the ${ }^{2} \mathrm{~T}_{1}$ state, and the longer component is assigned to the possible formation of ligand-porphyrin exciplex or the formation of dark charge-transfer states in the ${ }^{2} \mathrm{~T}_{1}$ state. ${ }^{36,37,46}$ Based on our ab initio calculations, we suggest another possible deactivation pathway. Porphyrin 6-Cu has a much lower ${ }^{2} \mathrm{~S}_{0} /{ }^{2} \mathrm{~S}_{1}$ gap, as the ${ }^{2} \mathrm{~S}_{1}$ state is located well below the Q band, and, therefore, direct IC is possible as an alternative deactivation channel. This view is also strongly supported by our experimental transient-absorption data. A shorter lifetime would then be interpreted as IC from the Soret band to the ${ }^{2} \mathrm{~S}_{1}$ state with no ISC involved, and a longer lifetime would correspond to IC from ${ }^{2} \mathrm{~S}_{1}$ to the ground state.

\section{Conclusion}

The meso-methylporphyrin derivatives introduced in this work possess several favorable properties for a PPG, including structural modularity, strong absorbance of light in the visible range of the spectrum, and high photouncaging efficiencies. The capacity to generate up to four productive photocleavable sites within a single molecule should enable the concomitant release of different leaving groups and could be especially valuable in the construction of lightresponsive polymer networks or hydrogels. ${ }^{47,48,49,50,51}$ The relatively high efficiency of ${ }^{1} \mathrm{O}_{2}$ generation exhibited by the porphyrin derivatives reported herein might be a limiting factor in some biological applications, where the photodynamic effect is detrimental, although several mitigation strategies are known. ${ }^{52,53,54,55,56}$ On the other hand, efficient ${ }^{1} \mathrm{O}_{2}$ generation can be synergistic to caging cytotoxic drugs. ${ }^{4,57}$

Importantly, the meso-methylporphyrin group represents a novel prototype of a PPG that unites traditionally exclusive properties of organic and metal-complex PPGs within a single structure. The functional separation between the metal binding chromophore and the site of leaving group release allows for an elective introduction of metal ions to manipulate its photophysical and photochemical properties. We expect that this approach could be implemented to repurpose additional known metal-binding ligands as PPGs, opening thus a new chemical space for the design of photocaged compounds with unique structural, chemical and photochemical properties. 


\section{Figures and Tables}

Figure 1: Meso-Methylporphyrin as a Hybrid Organic/Metal-Complex PPG Scaffold.

a State-of-the-art: organic PPGs OR metal-complex PPGs

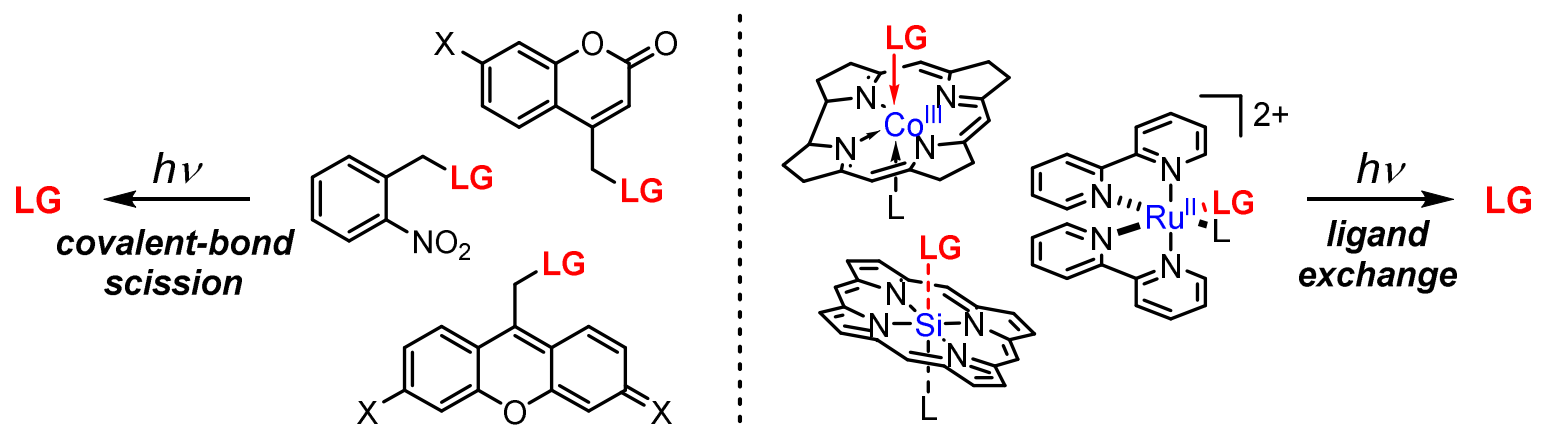

b This work: a hybrid organic/metal-complex PPG

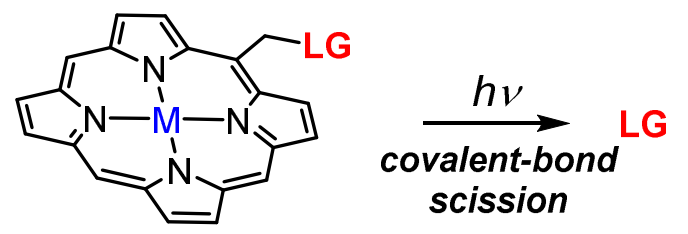

a, Contemporary organic and metal-complex PPGs; photorelease of leaving groups (LG) occurs via covalent bond scission or ligand exchange process, respectively. b, Mesomethylporphyrin as a hybrid organic/metal-complex PPG: diverse metal ion can be electively incorporated into the chromophore to modulate properties and a leaving group is photoreleased via covalent bond scission. 
Figure 2: Meso-Methylporphyrin as a Versatile PPG Scaffold: Synthesis and Photochemistry.
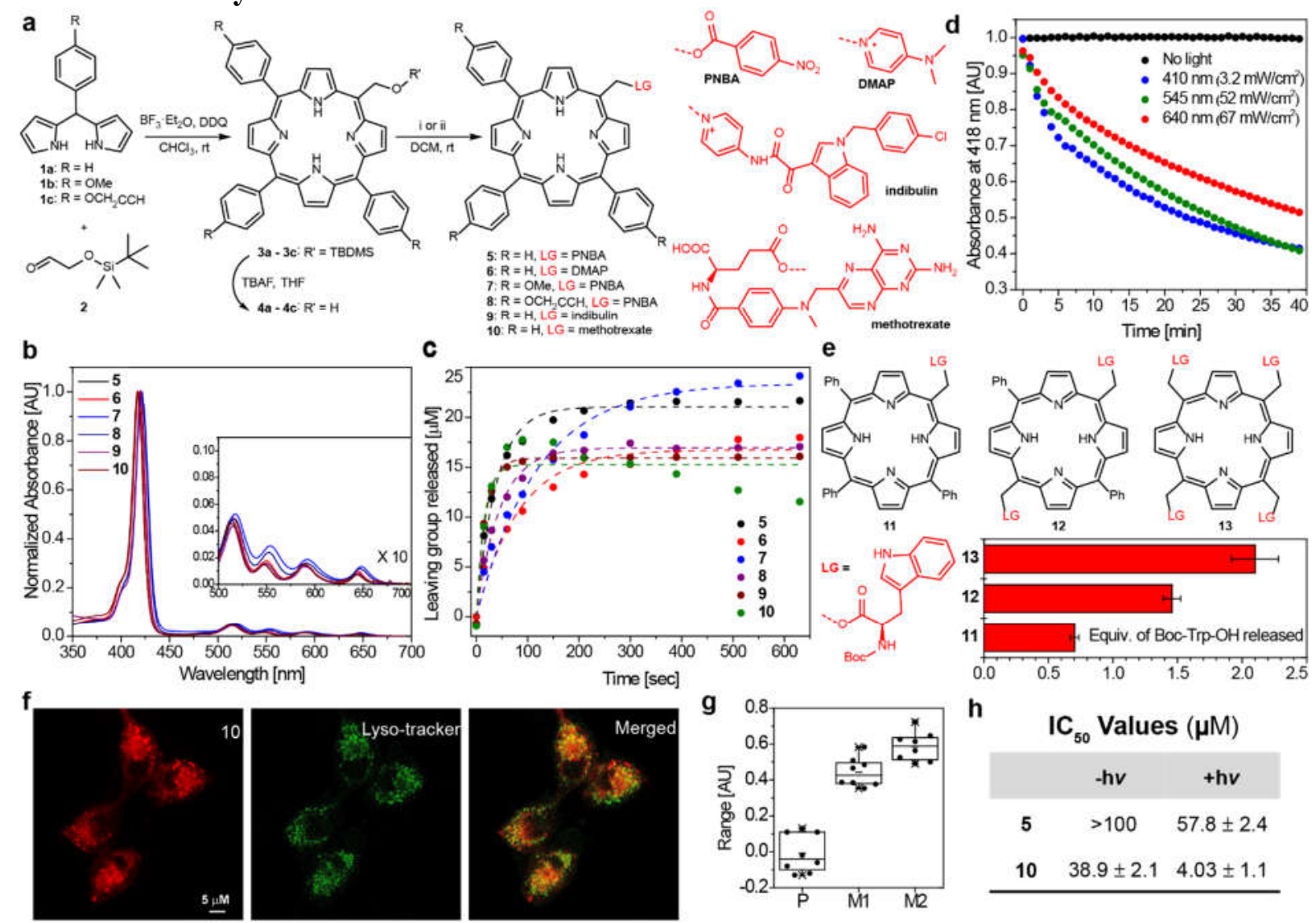

a, Synthesis of meso-methylporphyrin derivatives. i: PNBA or methotrexate, DMAP, DCC, 20 ${ }^{\circ} \mathrm{C}$. ii: mesylchloride, DMAP or indibulin, $20^{\circ} \mathrm{C}$. b, Absorption spectra of PNBA-, DMAP-, indibulin- and methotrexate-caged meso-methylporphyrins ( $3 \mu \mathrm{M}$, aerated DMSO). Inlet: a 10fold magnification of the Q-bands region. c, Photorelease of leaving groups from mesomethylporphyrin derivatives 5-10 $\left(25 \mu \mathrm{M}\right.$, degassed DMSO, $\lambda_{\mathrm{irr}}=410 \mathrm{~nm}, 40 \mathrm{~mW} \mathrm{~cm}{ }^{-2}$; HPLC-MS analysis). d, Photolysis of 6 at $\lambda_{\text {irr }}=410,545$ or $640 \mathrm{~nm}$. e, Structures of mono-, di- and tetra- $N_{\alpha}$-Boc-(L)-Trp caged meso-methylporphyrin derivatives (11-13) and photorelease quantification of leaving groups photoreleased $\left(25 \mu \mathrm{M}\right.$, degassed DMSO, $\lambda_{\text {irr }}=$ $410 \mathrm{~nm}, 40 \mathrm{~mW} \mathrm{~cm}^{-2}$, HPLC-MS analysis). Averages from three experiments are shown; error bars represent the standard deviation. $\mathbf{f}$, Representative fluorescence microscopy images of the cellular distribution of $\mathbf{1 0}$ in cultured murine 4T1 mammary carcinoma cells. Red: compound 10, green: LysoTracker Green. $\mathbf{g}$, Co-localization parameters of $\mathbf{1 0}$ and LysoTracker Green. $\mathbf{h}$, Calculated $\mathrm{IC}_{50}$ concentrations of $\mathbf{5}$ and $\mathbf{1 0}$ in cultured murine 4T1 mammary carcinoma cells following or not irradiation with $545 \mathrm{~nm}$ light for $5 \mathrm{~min}$. The experiment was repeated at least twice in triplicates. 
Table 1. Photophysical and Photochemical Properties of meso-Methylporphyrin Derivatives.

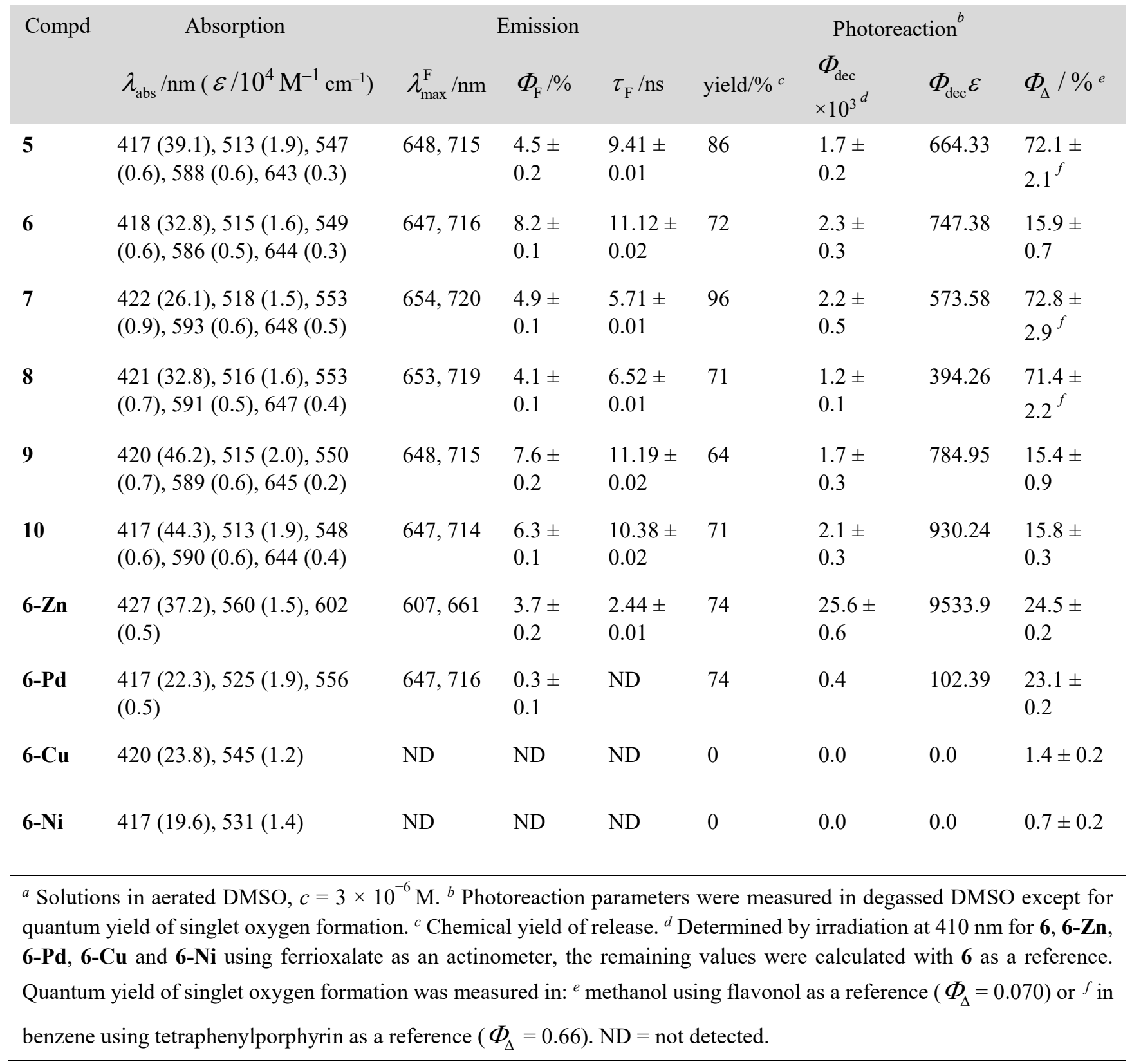


Figure 3. Metal-Containing Meso-Methylporphyrin PPGs: Synthesis and Photochemistry.

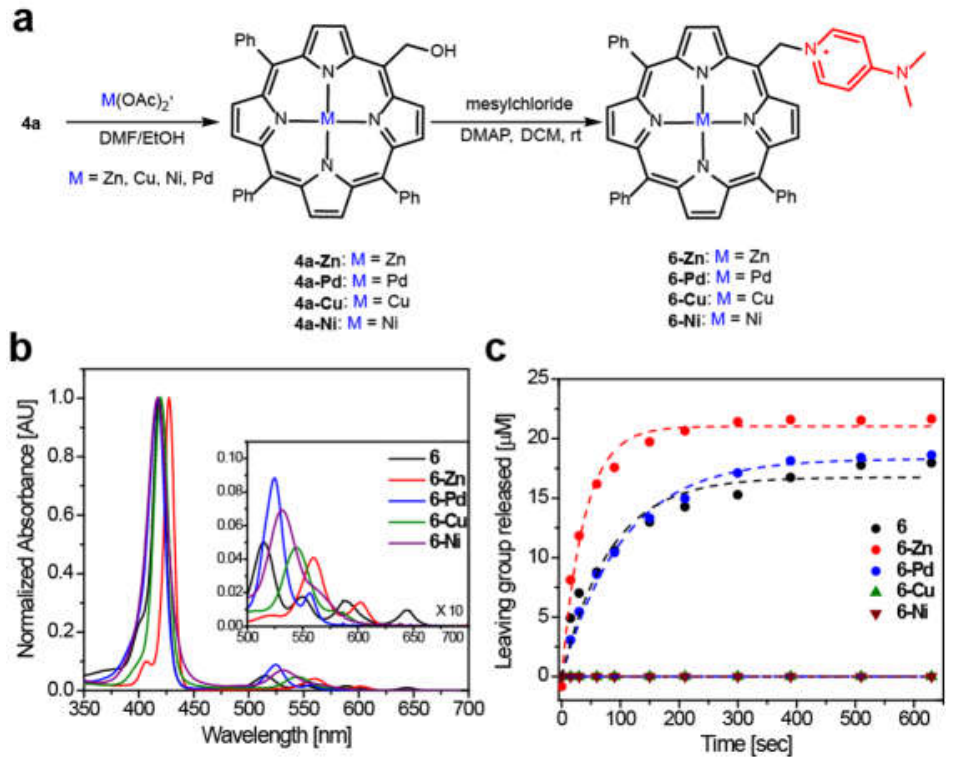

a, Synthesis of metal-containing meso-methylporphyrins followed by installation of DMAP as a model leaving group. b, Absorption spectra of DMAP-caged metal-containing mesomethylporphyrin derivatives ( $3 \mu \mathrm{M}$, aerated DMSO); that of an analogous metal-free mesomethylporphyrin (6) is shown for comparison. Inlet: a 10-fold magnification of the Q-bands region. c, Photorelease of DMAP from metal-containing meso-methylporphyrin derivatives (25 $\mu \mathrm{M}$, degassed DMSO, $\lambda_{\text {irr }}=410 \mathrm{~nm}, 40 \mathrm{~mW} \mathrm{~cm}^{-2}$; HPLC-MS analysis), that of an analogous metal-free meso-methylporphyrin (6) is included for comparison. 
Table 2. Major Photophysical and Photochemical Processes Observed in 6, 6-Zn, and 6Pd.

\begin{tabular}{|c|c|c|c|c|c|c|c|c|c|}
\hline \multirow{3}{*}{$\begin{array}{c}\text { Compd } \\
\quad a\end{array}$} & \multirow{3}{*}{$k_{\mathrm{Q}}^{1} / \mathrm{s}^{-1 b}$} & \multirow{3}{*}{$k_{\mathrm{Q}}^{2} / \mathrm{s}^{-1 b}$} & \multirow{3}{*}{$k_{\mathrm{ISC}} / \mathrm{s}^{-1 c}$} & \multicolumn{4}{|c|}{$k_{\mathrm{d}} / \mathrm{s}^{-1 d}$} & \multirow{3}{*}{$\Phi_{\mathrm{F}}^{\mathrm{f}}$} & \multirow{3}{*}{$\begin{array}{c}\tau_{\mathrm{F}} / \mathrm{ns} \\
{ }_{g}\end{array}$} \\
\hline & & & & \multicolumn{2}{|c|}{ DMSO } & \multicolumn{2}{|c|}{ Methanol } & & \\
\hline & & & & Aerated & Degassed & Aerated & Degassed & & \\
\hline 6 & $4.1 \times 10^{10}$ & $3.7 \times 10^{9}$ & $1.7 \times 10^{8}$ & $6.3 \times 10^{5}$ & $9.1 \times 10^{4}$ & $3.3 \times 10^{6}$ & $1.7 \times 10^{5}$ & 0.082 & 11.12 \\
\hline $6-\mathrm{Zn}$ & $6.9 \times 10^{10}$ & $8.0 \times 10^{9}$ & $2.2 \times 10^{8}$ & $4.0 \times 10^{5}$ & $6.0 \times 10^{4}$ & $2.1 \times 10^{6}$ & $9.4 \times 10^{4}$ & 0.037 & 2.44 \\
\hline 6-Pd & ND & ND & $1.6 \times 10^{10}$ & $6.7 \times 10^{5}$ & $1.1 \times 10^{5}$ & $3.2 \times 10^{6}$ & $6.6 \times 10^{5}$ & 0.0034 & ND \\
\hline 6-Cu & ND & ND & ND & $2.9 \times 10^{10}$ & & & & ND & ND \\
\hline
\end{tabular}

${ }^{a}$ fs-TA spectroscopy: $\lambda_{\text {exc }}=387 \mathrm{~nm}$; concentrations of $\sim 0.2-0.4 \mathrm{mM}$; ns-TA spectroscopy: $\lambda_{\text {exc }}=355 \mathrm{~nm}$; concentrations $6-18 \mu \mathrm{M}) ;{ }^{b}$ The rate constants of transitions within the Q states; ${ }^{c}$ intersystem crossing $\left(k_{\text {ISC }}\right) ;{ }^{d}$ triplet-state decay $\left(k_{\mathrm{d}}\right)$; ${ }^{e}$ decay in an aerated DMSO solution. ${ }^{f} \Phi_{\mathrm{F}}$ are the fluorescence quantum yields, and ${ }^{g} \tau_{\mathrm{F}}$ are the fluorescence lifetimes determined in DMSO. ND = not determined. 
Figure 4. Mechanistic Details of the Photochemistry of DMAP-Caged Porphyrin Derivatives.
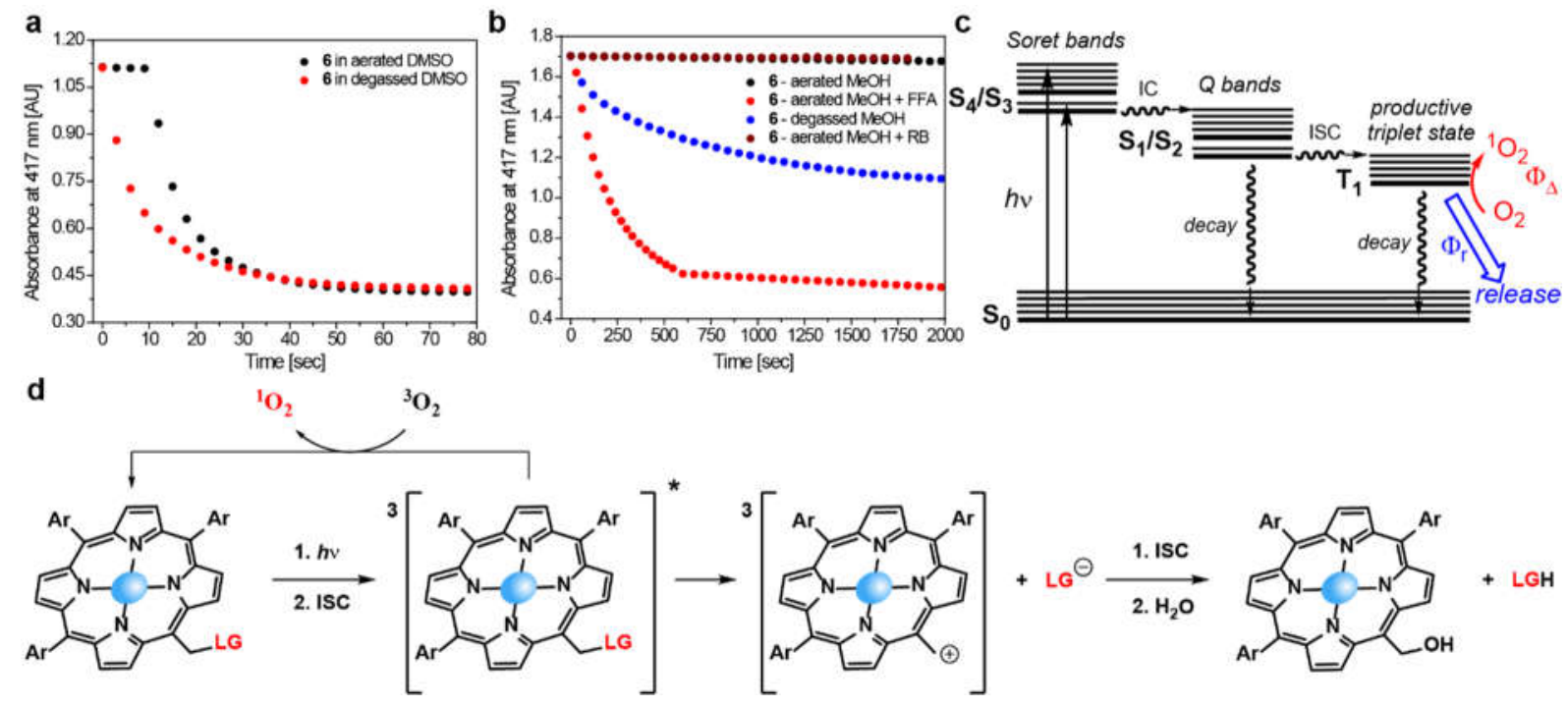

a, Photochemistry of $\mathbf{6}$ in aerated or degassed DMSO solutions. b, Photochemistry of $\mathbf{6}$ in aerated or degassed methanol solutions $\left(\lambda_{\text {irr }}=420 \mathrm{~nm}\right)$ and in the presence of a singlet oxygen generator (rose bengal, RB, $\lambda_{\text {irr }}=545 \mathrm{~nm}$ ) or quencher (furfuryl alcohol, FFA). c, A general Jablonski diagram. d, The proposed photorelease mechanism for porphyrin PPGs. 


\section{Acknowledgments}

R.W. acknowledges support from the European Research Council (GAtransport). Support for this work was provided by the Czech Science Foundation (GA21-01799S), and we thank the CETOCOEN EXCELLENCE Teaming 2 project (supported by the Czech Ministry of Education, Youth, and Sports: CZ.02.1.01/0.0/0.0/17_043/0009632 and EU H2020: 857560) and the RECETOX research infrastructure (LM2018121) (P. K.). This work was supported by JSPS KAKENHI Grant Number JP18J20500 (Y. C.). P. S. and J. J. thank the Czech Science Foundation, project No. 20-15825S. A. R. S. thanks the Global Research and Training Fellowship (GRTF) from Tel Aviv University.

\section{Contributions}

A.R.S. and R.W. conceived the project and designed the initial experiments. A.R.S. and A.R. performed the synthesis and characterization of the metal complexes and the organic compounds and performed photophysical measurements under the supervision of R.W. and P.K. A.R.S., Y.C., and A.R. designed and performed the experimental mechanistic investigation under the supervision of P.K. J.J. designed and performed the quantum-chemical calculations under the supervision of P.S. Cellular studies were designed and performed by A.R.S. and S.I.D. under the supervision of R.S.F. The Manuscript was written by A.R.S., P.K. and R.W. All authors analyzed the data, discussed the results and commented on the manuscript.

\section{Competing interests}

The authors declare no competing interests.

\section{Data availability}

All the data generated or analyzed during this study are included in this article and its Supplementary Information. Crystallographic data have been deposited at the Cambridge Crystallographic Data Centre (CCDC) as 1973540 and can be obtained free of charge from the CCDC via www.ccdc.cam.ac.uk/getstructures.

\section{References}

1. Zhao, H., Sterner, E. S., Coughlin, E. B., Theato, P. o-Nitrobenzyl Alcohol Derivatives: Opportunities in Polymer and Materials Science. Macromolecules 45, 1723-1736 (2012).

2. Ellis-Davies, G. C. R. Useful Caged Compounds for Cell Physiology. Acc. Chem. Res. 53, 1593-1604 (2020).

3. Ruskowitz, E. R., DeForest, C. A. Photoresponsive Biomaterials for Targeted Drug Delivery and 4D Cell Culture. Nat. Rev. Mater. 3, 17087 (2018). 
4. Silva, J. M., Silva, E., Reis, R. L. Light-triggered release of photocaged therapeutics Where are we now? J. Controlled Release 298, 154-176 (2019).

5. Barltrop, J. A., Schofield, P. Photosensitive Protecting Groups. Tetrahedron Lett. 3, 697-699 (1962).

6. Barton, D. H. R., Chow, Y. L., Cox, A., Kirby, G. W. Photosensitive protection of functional groups. Tetrahedron Lett. 3, 1055-1057 (1962).

7. Klán, P., Šolomek, T., Bochet, C. G., Blanc, A., Givens, R., Rubina, M., et al. Photoremovable Protecting Groups in Chemistry and Biology: Reaction Mechanisms and Efficacy. Chem. Rev. 113, 119-191 (2013).

8. Weinstain, R., Slanina, T., Kand, D., Klán, P. Visible-to-NIR-Light Activated Release: From Small Molecules to Nanomaterials. Chem. Rev. 120, 13135-13272 (2020).

9. Wang, X., Wang, X., Jin, S., Muhammad, N., Guo, Z. Stimuli-Responsive Therapeutic Metallodrugs. Chem. Rev. 119, 1138-1192 (2019).

10. Renfrew, A. K. Transition metal complexes with bioactive ligands: mechanisms for selective ligand release and applications for drug delivery. Metallomics 6, 1324-1335 (2014).

11. Goswami, P. P., Syed, A., Beck, C. L., Albright, T. R., Mahoney, K. M., Unash, R., et al. BODIPY-Derived Photoremovable Protecting Groups Unmasked with Green Light. J. Am. Chem. Soc. 137, 3783-3786 (2015).

12. Rubinstein, N., Liu, P., Miller, E. W., Weinstain, R. meso-Methylhydroxy BODIPY: a scaffold for photo-labile protecting groups. Chem. Commun. 51, 6369-6372 (2015).

13. Šolomek, T., Wirz, J., Klán, P. Searching for Improved Photoreleasing Abilities of Organic Molecules. Acc. Chem. Res. 48, 3064-3072 (2015).

14. Arsenault, G. P., Bullock, E., MacDonald, S. F. Pyrromethanes and Porphyrins Therefrom1. J. Am. Chem. Soc. 82, 4384-4389 (1960).

15. Littler, B. J., Ciringh, Y., Lindsey, J. S. Investigation of Conditions Giving Minimal Scrambling in the Synthesis of trans-Porphyrins from Dipyrromethanes and Aldehydes. J. Org. Chem. 64, 2864-2872 (1999).

16. Geier Iii, G. R., Littler, B. J., Lindsey, J. S. Investigation of porphyrin-forming reactions. Part 3.1 The origin of scrambling in dipyrromethane + aldehyde 
condensations yielding trans-A2B2-tetraarylporphyrins. J. Chem. Soc., Perkin Trans. 2, 701-711 (2001).

17. Gouterman, M., Wagnière, G. H., Snyder, L. C. Spectra of porphyrins: Part II. Four orbital model. J. Mol. Spectrosc. 11, 108-127 (1963).

18. Gouterman, M. Spectra of porphyrins. J. Mol. Spectrosc. 6, 138-163 (1961).

19. Mandal, A. K., Taniguchi, M., Diers, J. R., Niedzwiedzki, D. M., Kirmaier, C., Lindsey, J. S., et al. Photophysical Properties and Electronic Structure of Porphyrins Bearing Zero to Four meso-Phenyl Substituents: New Insights into Seemingly Well Understood Tetrapyrroles. J. Phys. Chem. A 120, 9719-9731 (2016).

20. Thomas, D. W., Martell, A. E. Absorption Spectra of para-Substituted Tetraphenylporphines1,2. J. Am. Chem. Soc. 78, 1338-1343 (1956).

21. Inokuma, Y., Yoon, Z. S., Kim, D., Osuka, A. meso-Aryl-Substituted Subporphyrins: Synthesis, Structures, and Large Substituent Effects on Their Electronic Properties. $J$. Am. Chem. Soc. 129, 4747-4761 (2007).

22. Tang, X.-J., Wu, Y., Zhao, R., Kou, X., Dong, Z., Zhou, W., et al. Photorelease of Pyridines Using a Metal-Free Photoremovable Protecting Group. Angew. Chem. Int. Ed. 59, 18386-18389 (2020).

23. Slanina, T., Shrestha, P., Palao, E., Kand, D., Peterson, J. A., Dutton, A. S., et al. In Search of the Perfect Photocage: Structure-Reactivity Relationships in meso-Methyl BODIPY Photoremovable Protecting Groups. J. Am. Chem. Soc. 139, 15168-15175 (2017).

24. Chitose, Y., Abe, M., Furukawa, K., Lin, J.-Y., Lin, T.-C., Katan, C. Design and Synthesis of a Caged Carboxylic Acid with a Donor- $\pi-$ Donor Coumarin Structure: One-photon and Two-photon Uncaging Reactions Using Visible and Near-Infrared Lights. Org. Lett. 19, 2622-2625 (2017).

25. Ion, R.-M. Porphyrins and phthalocyanines: photosensitizers and photocatalysts. Phthalocyanines and Some Current Applications 189-221 (2017).

26. Ostapko, J., Gorski, A., Buczyńska, J., Golec, B., Nawara, K., Kharchenko, A., et al. Towards More Photostable, Brighter, and Less Phototoxic Chromophores: Synthesis and Properties of Porphyrins Functionalized with Cyclooctatetraene. Chem. Eur. J. 26, 16666-16675 (2020). 
27. Lutkus, L. V., Rickenbach, S. S., McCormick, T. M. Singlet oxygen quantum yields determined by oxygen consumption. Journal of Photochemistry and Photobiology A 378, 131-135 (2019).

28. Wan, S., Lu, W. Reversible Photoactivated Phosphorescence of Gold(I) Arylethynyl Complexes in Aerated DMSO Solutions and Gels. Angew. Chem. Int. Ed. 56, 17841788 (2017).

29. Kumar, P. H., Venkatesh, Y., Siva, D., Ramakrishna, B., Bangal, P. R. Ultrafast relaxation dynamics of 5,10,15,20- meso- tetrakis pentafluorophenyl porphyrin studied by fluorescence up-conversion and transient absorption spectroscopy. J. Phys. Chem. A 119, 1267-1278 (2015).

30. Harriman, A. Luminescence of Porphyrins and Metalloporphyrins. J. Chem. Soc. 77, 1281-1291 (1981).

31. Kee, H. L., Bhaumik, J., Diers, J. R., Mroz, P., Hamblin, M. R., Bocian, D. F., et al. Photophysical characterization of imidazolium-substituted $\mathrm{Pd}(\mathrm{II}), \mathrm{In}(\mathrm{III})$, and $\mathrm{Zn}$ (II) porphyrins as photosensitizers for photodynamic therapy. J. Photochem. Photobiol. A 200, 346-355 (2008).

32. Kim, D., Holten, D., Gouterman, M., Buchler, J. W. Evidence From Picosecond Transient Absorption and Kinetic Studies of Charge-Transfer States in Copper(II) Porphyrins. J. Am. Chem. Soc. 106, 2793-2798 (1984).

33. Kobayashi, T., Straub, K. D., Rentzepis, P. M. Energy Relaxation Mechanism in Ni(II), Pd(II), Pt(II) and Zn(II) Porphyrins. Photochem. Photobiol. 29, 925-931 (1979).

34. Obondi, C. O., Lim, G. N., D'Souza, F. Triplet-triplet excitation transfer in palladium porphyrin-fullerene and platinum porphyrin-fullerene dyads. J. Phys. Chem. C 119, 176-185 (2015).

35. Ohno, O., Kaizu, Y., Kobayashi, H. Luminescence of some metalloporphins including the complexes of the IIIb metal group. The Journal of Chemical Physics 82, 1779-1787 (1985).

36. Jeoung, S. C., Takeuchi, S., Tahara, T., Kim, D. Ultrafast decay dynamics of photoexcited $\mathrm{Cu}(\mathrm{II})(\mathrm{TMpy}-\mathrm{P} 4)$ in water solvent. Chem. Phys. Lett. 309, 369-376 (1999).

37. Jeong, D., Kang, D.-g., Joo, T., Kim, S. K. Femtosecond-Resolved Excited State Relaxation Dynamics of Copper (II) Tetraphenylporphyrin (CuTPP) After Soret Band Excitation. Sci. Rep. 7, 16865 (2017). 
38. Asano, M., Kaizu, Y., Kobayashi, H. The lowest excited states of copper porphyrins. $J$. Chem. Phys. 89, 6567-6576 (1988).

39. Harriman, A. Luminescence of porphyrins and metalloporphyrins. Part 3.- Heavyatom effects. Journal of the Chemical Society, Faraday Transactions 77, 1281-1291 (1981).

40. Kee, H. L., Bhaumik, J., Diers, J. R., Mroz, P., Hamblin, M. R., Bocian, D. F., et al. Photophysical Characterization of Imidazolium-Substituted Pd(II), In(III), and Zn(II) Porphyrins as Photosensitizers for Photodynamic Therapy. J. Photochem. Photobiol., A 200, 346-355 (2008).

41. Obondi, C. O., Lim, G. N., D’Souza, F. Triplet-Triplet Excitation Transfer in Palladium Porphyrin-Fullerene and Platinum Porphyrin-Fullerene Dyads. Journal of Physical Chemistry C 119, 176-185 (2015).

42. Ohno, O., Kaizu, Y., Kobayashi, H. Luminescence of some metalloporphins including the complexes of the IIIb metal group. J. Chem. Phys. 82, 1779-1787 (1985).

43. Moroni, L., Gellini, C., Salvi, P. R., Marcelli, A., Foggi, P. Excited States of Porphyrin Macrocycles. J. Phys. Chem. A 112, 11044-11051 (2008).

44. Perun, S., Tatchen, J., Marian, C. M. Singlet and Triplet Excited States and Intersystem Crossing in Free-Base Porphyrin: TDDFT and DFT/MRCI Study. ChemPhysChem 9, 282-292 (2008).

45. Jeong, D., Kang, D. G., Joo, T., Kim, S. K. Femtosecond-Resolved Excited State Relaxation Dynamics of Copper (II) Tetraphenylporphyrin (CuTPP) after Soret Band Excitation. Sci. Rep. 7, 1-8 (2017).

46. Chirvony, V. S., Négrerie, M., Martin, J.-L., Turpin, P.-Y. Picosecond Dynamics and Mechanisms of Photoexcited $\mathrm{Cu}(\mathrm{II})-5,10,15,20$-meso-tetrakis(4-Nmethylpyridyl)porphyrin Quenching by Oxygen-Containing Lewis-Base Solvents. $J$. Phys. Chem. A 106, 5760-5767 (2002).

47. Rapp, T. L., DeForest, C. A. Visible Light-Responsive Dynamic Biomaterials: Going Deeper and Triggering More. Adv. Healthc. Mater. 9, 1901553 (2020).

48. Kloxin, A. M., Kasko, A. M., Salinas, C. N., Anseth, K. S. Photodegradable Hydrogels for Dynamic Tuning of Physical and Chemical Properties. Science 324, 59 (2009).

49. LeValley, P. J., Neelarapu, R., Sutherland, B. P., Dasgupta, S., Kloxin, C. J., Kloxin, A. M. Photolabile Linkers: Exploiting Labile Bond Chemistry to Control Mode and 
Rate of Hydrogel Degradation and Protein Release. J. Am. Chem. Soc. 142, 4671-4679 (2020).

50. Raman, R., Hua, T., Gwynne, D., Collins, J., Tamang, S., Zhou, J., et al. Lightdegradable hydrogels as dynamic triggers for gastrointestinal applications. Sci. Adv. 6, eaay0065 (2020).

51. Huynh, C. T., Nguyen, M. K., Tonga, G. Y., Longé, L., Rotello, V. M., Alsberg, E. Photocleavable Hydrogels for Light-Triggered siRNA Release. Adv. Healthc. Mater. 5, 305-310 (2016).

52. Hirakawa, K., Harada, M., Okazaki, S., Nosaka, Y. Controlled generation of singlet oxygen by a water-soluble meso-pyrenylporphyrin photosensitizer through interaction with DNA. Chem. Commun. 48, 4770-4772 (2012).

53. Hirakawa, K., Nishimura, Y., Arai, T., Okazaki, S. Singlet Oxygen Generating Activity of an Electron Donor Connecting Porphyrin Photosensitizer Can Be Controlled by DNA. J. Phys. Chem. B 117, 13490-13496 (2013).

54. Jeong, H.-G., Choi, M.-S. Design and Properties of Porphyrin-based Singlet Oxygen Generator. Isr. J. Chem. 56, 110-118 (2016).

55. Mathai, S., Smith, T. A., Ghiggino, K. P. Singlet oxygen quantum yields of potential porphyrin-based photosensitisers for photodynamic therapy. Photochem. Photobiol. Sci. 6, 995-1002 (2007).

56. McCarthy, J. R., Weissleder, R. Model Systems for Fluorescence and Singlet Oxygen Quenching by Metalloporphyrins. ChemMedChem 2, 360-365 (2007).

57. Bonnet, S. Why develop photoactivated chemotherapy? Dalton Trans. 47, 1033010343 (2018). 